\title{
What are the underlying units of perceived animacy? Chasing detection is intrinsically object-based
}

\author{
Benjamin van Buren $^{1} \cdot$ Tao Gao $^{2} \cdot$ Brian J. Scholl $^{1}$
}

Published online: 3 February 2017

(C) Psychonomic Society, Inc. 2017

\begin{abstract}
One of the most foundational questions that can be asked about any visual process is the nature of the underlying 'units' over which it operates (e.g., features, objects, or spatial regions). Here we address this question - for the first time, to our knowledge - in the context of the perception of animacy. Even simple geometric shapes appear animate when they move in certain ways. Do such percepts arise whenever any visual feature moves appropriately, or do they require that the relevant features first be individuated as discrete objects? Observers viewed displays in which one disc (the "wolf") chased another (the "sheep") among several moving distractor discs. Critically, two pairs of discs were also connected by visible lines. In the Unconnected condition, both lines connected pairs of distractors; but in the Connected condition, one connected the wolf to a distractor, and the other connected the sheep to a different distractor. Observers in the Connected condition were much less likely to describe such displays using mental state terms. Furthermore, signal detection analyses were used to explore the objective ability to discriminate chasing displays from inanimate control displays in which the wolf moved toward the sheep's mirror-image. Chasing detection was severely impaired on Connected trials: observers could readily detect an object chasing another object, but not a line-end chasing another line-end, a line-end chasing an object, or an object chasing a line-end. We conclude that the
\end{abstract}

Brian J. Scholl

brian.scholl@yale.edu

Benjamin van Buren

benjamin.vanburen@yale.edu

1 Department of Psychology, Yale University, Box 208205, New Haven, CT 06520-8205, USA

2 Massachusetts Institute of Technology, Cambridge, MA, USA underlying units of perceived animacy are discrete visual objects.

Keywords Animacy $\cdot$ Intentionality $\cdot$ Chasing $\cdot$ Object-based attention

Visual processing traffics not only in low-level features, such as color and orientation, but also in properties that we typically associate with higher-level cognition, such as animacy and intentionality. For example, even simple geometric shapes are reflexively seen as animate and goal-directed when they move in certain ways (Heider \& Simmel, 1944; Michotte, 1950/1991; for a review, see Scholl \& Tremoulet, 2000). Such phenomena seem to reflect visual processing (and not just higher level judgment), as they are tightly constrained by subtle features of the visual input, while proving immune to observers' explicit goals and beliefs (for a review, see Scholl \& Gao, 2013). Indeed, visuomotor behavior is influenced by perceived animacy even when observers actively try to ignore such features (van Buren, Uddenberg, \& Scholl, 2016). In addition - and like other aspects of visual processing - the perception of animacy appears to be a universal feature of human experience, arising early in typical development (e.g., Csibra, 2008), and manifesting similarly in individuals from different cultures (e.g., Barrett, Todd, Miller, \& Blythe, 2005).

Despite decades of scientific exploration, some surprisingly fundamental questions about perceived animacy remain open. For example, relatively little work has explored how this phenomenon interacts with other aspects of perception and cognition (see Gao, McCarthy, \& Scholl, 2010; van Buren \& Scholl, in press), and no previous research to our knowledge has explored the underlying 'units' over which this form of perception operates. It thus seems exciting and worthwhile to explore whether perceived animacy may be 
integrated with other forms of perception, by being constrained by the same sorts of object-based limits.

\section{Object-based visual cognition}

Just as functions in a computer program may operate over only certain types of data structures, so too may visual processes operate over only certain types of underlying representations. Accordingly, a great deal of research has been devoted to figuring out the underlying 'units' of various processeswhere candidates include units such as individual visual features, continuous spatial regions of the visual field, or discrete visual objects. And in many cases, the underlying units of visual processing have turned out to be discrete objects. This conclusion has been suggested, for example, in what may be the two most prominent areas of research in visual cognition-attention and visual working memory.

In the study of visual attention, researchers once characterized the units of selection in terms of spatial extent - as if attention operated as a kind of spotlight or zoom-lens (for a review, see Cave \& Bichot, 1999). Subsequent work revealed, however, that the strength of attentional selection was mediated by the structure of visual scenes, controlling for spatial extent. Such phenomena of object-based attention (for reviews, see Chen, 2012; Scholl, 2001) revealed that attention more readily selects multiple regions when they lie within the same (preattentively parsed) visual object (e.g., Egly, Driver, \& Rafal, 1994), where such objects are parsed on the basis of cues such as connectedness and closure (e.g., Feldman, 2007; Marino \& Scholl, 2005). Indeed, simply connecting two shapes with a thin line can effectively frustrate the ability to select only one of them (Scholl, Pylyshyn, \& Feldman, 2001). In this sense, the underlying units of attention often appear to be discrete objects rather than spatial regions.

In the study of visual working memory, researchers have contrasted discrete objects not with spatial regions but with individual visual features. For example, it has been argued that the capacity of visual working memory is limited not by the number of features (e.g., colors or orientations) in a display, but rather by the number of objects on which those features appear (for a review, see Luck \& Vogel, 2013) - and in the limit it has been argued that you can just as readily remember 16 visual features as you can four features, as long as the 16 features are distributed across only four objects (Luck \& Vogel, 1997; Vogel, Woodman, \& Luck, 2001; for a summary of the debate involving such claims, see Suchow, Fougnie, Brady, \& Alvarez, 2014).

\section{The current project: the units of perceived chasing}

As with attention and memory, we can ask: What are the underlying units of perceived animacy? To investigate this, we needed a simple and robust cue to animacy, and we settled on chasing. Chasing has obvious ecological significance, and even in modern life the single most commonly remembered dream is that of being chased (Garfield, 2001; Revonsuo, 2000). Infants attend to chasing early in development (Rochat, Morgan, \& Carpenter, 1997), and adult observers are strikingly sensitive to chasing, over and above factors such as correlated motion and proximity, as when one disc (the "wolf") continually updates its heading in the direction of another disc (the "sheep"; Dittrich \& Lea, 1994; Gao, Newman, \& Scholl, 2009; Gao \& Scholl, 2011).

In this project, observers viewed displays in which a wolf disc moved toward a sheep disc among moving distractors (see Fig. 1a), but in some conditions we disrupted the objecthood of the wolf and sheep by connecting them to different distractors with thin lines (see Fig. 1b). If the perception of animacy is intrinsically object-based, then connecting the wolf and sheep to distractors should impair the perception of chasing - in that it should be harder to detect a clearly visible line-end chasing another clearly visible line-end, compared to one object chasing another. Otherwise, if the perception of chasing operates just as efficiently over any discriminable visual feature, then this manipulation should make no difference - since (a) the individual discs are still easily perceived despite the thin lines, (b) the lines themselves are included in all displays, and (c) this manipulation is made completely independently of all other features of the displays (such as the locations and motions of the discs) that otherwise determine the perception of chasing (e.g., Meyerhoff, Schwan, \& Huff, 2014).

Of course, such displays are difficult to depict in static figures, but dynamic animations of all displays described below can be viewed online (http://perception.yale.edu/objectbased-chasing/) .

\section{Experiment 1: phenomenology}

In an initial experiment, we were interested in whether observers would experience and describe chasing events differently depending on whether the wolf and the sheep were discrete objects. Each observer viewed an animation in which a wolf disc chased a sheep disc. In the Connected condition, the wolf and sheep were each connected to other moving distractor discs with thin lines (see Fig. 1b) - a simple manipulation that reliably influences whether shapes such as the discs are perceived as individual objects (e.g., Fornaciai, Cicchini, \& Burr, 2016; Franconeri, Bemis, \& Alvarez, 2009; He, Zhang, Zhou, \& Chen, 2009; Howe, Incledon, \& Little, 2012; Scholl et al., 2001). In the Unconnected condition, the display contained identically drawn lines, which connected pairs of distractors instead of involving the wolf or sheep. Observers simply viewed one of these displays and 


\section{A Unconnected}
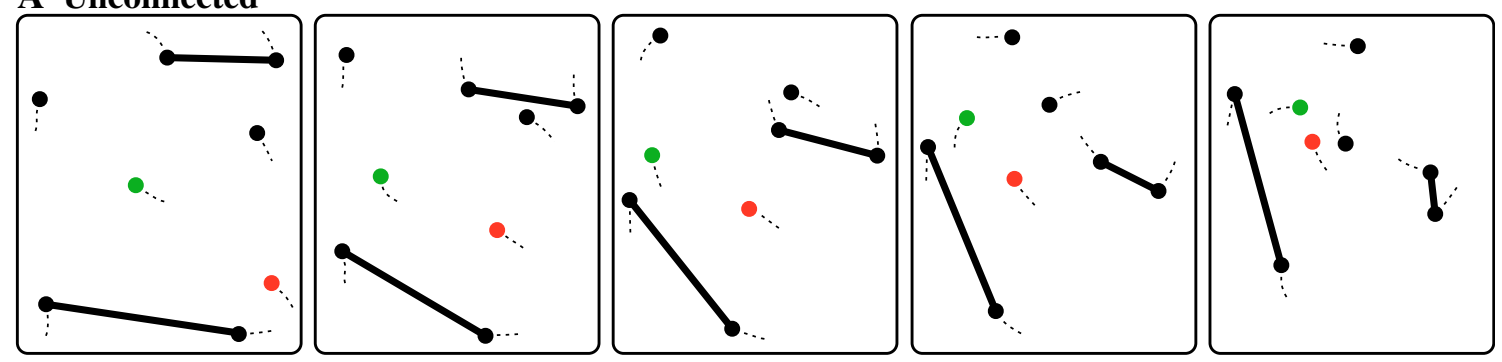

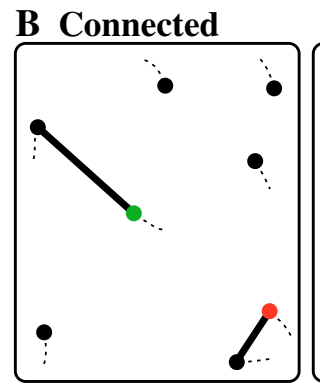

T1

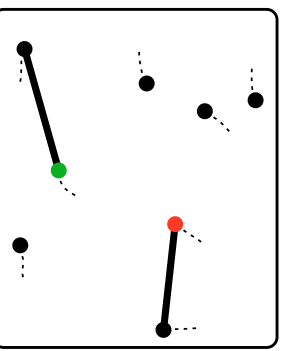

T2

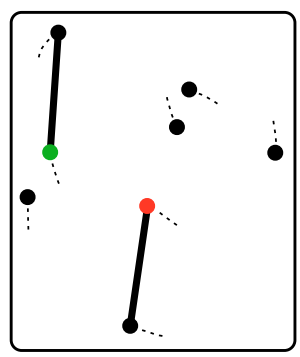

T3

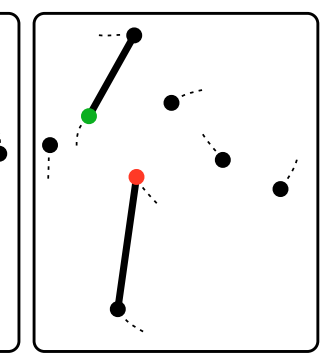

T4

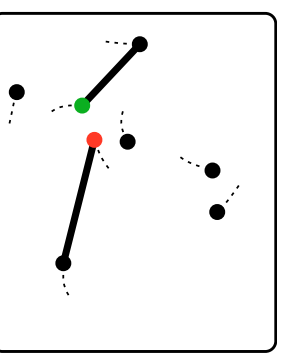

T5
Fig. 1 a Sequence of frames depicting Unconnected condition in Experiments $1-3$. The wolf (depicted in red) continually updates its heading to approach the sheep (depicted in green). The other (distractor) discs move randomly, and two pairs of these discs
Connected thin lines. b Sequence of frames Connected condition in Experiments 1 and 2. Here, all display elements move identically to the Unconnected condition, but the lines instead connect the wolf and sheep to two different distractors (Color figure online) then described it - and we evaluated how the connectedness manipulation influenced the use of mental state language in the resulting descriptions.

\section{Method}

Observers Eighty observers (with normal or corrected-tonormal acuity) - 40 in each of the two conditions - participated in 2-min sessions in exchange for candy. This sample size was based on a power analysis run on pilot data.

Apparatus Stimuli were presented using custom software written in Python with the PsychoPy libraries (Peirce, 2007). Observers sat without restraint approximately $60 \mathrm{~cm}$ from the display, which subtended approximately $43.19^{\circ} \times 27.79^{\circ}$ (with all extents below reported based on this distance).

Stimuli Animations contained eight $.39^{\circ}$ white discs-a wolf, a sheep, and six distractors-which moved at $6.47^{\circ} / \mathrm{s}$ within a $15.56^{\circ}$ square region at the display's center. This region had a white $\left(.27^{\circ}\right)$ border, which separated its black interior from the black surround, and discs initially appeared in randomized positions fully within the square, with the wolf and sheep at least $10.41^{\circ}$ apart. The initial heading of each disc was randomized. On each frame of motion, the sheep and distractor discs each had a $10 \%$ chance of assuming a new randomized heading within a $90^{\circ}$ window (centered on its current heading), and the wolf had a $10 \%$ chance of updating its heading in the direction of the sheep. Each disc thus updated its trajectory roughly every $333 \mathrm{~ms}$. Whenever a disc reached the border, it "bounced" in order to stay within bounds. The display also contained two $.19^{\circ}$ white lines that changed position and length to continuously connect the centers of two pairs of discs: in the Unconnected condition (see Fig. 1a) the lines connected randomly chosen pairs of distractors, and in the Connected condition (see Fig. 1b), they connected the wolf and sheep to different randomly chosen distractors.

Procedure Observers were told that they would view a short animation containing moving shapes, that two of these would be highlighted at the start, and that they should pay special attention to these shapes' movements so as to later be able to describe their relationship. For the first $3 \mathrm{~s}$, all shapes were static and the wolf and sheep were surrounded by flashing green outlines $\left(.16^{\circ}\right.$ thick; $167 \mathrm{~ms}$ on, $167 \mathrm{~ms}$ off; these were drawn on top of any overlapping shapes). The outlines then disappeared and all shapes moved for $15 \mathrm{~s}$. Afterwards, observers typed a " $1-2$ sentence description" of "the relationship between the two highlighted shapes."

\section{Results and discussion}

A rater coded observers' descriptions based on whether they used mental state language such as "chasing" or "following." 
Descriptions were then coded by a different rater (interrater agreement/Cohen's kappa $=.95$ ). The resulting rates of mental state language use for observers in the Unconnected and Connected conditions are depicted in Fig. 2. Inspection of this figure suggests that observers in the Unconnected condition were twice as likely to use mental state terms as were observers in the Connected condition, and this impression was verified with a chi-square test, $\chi^{2}(1, N=80)=7.27, p=.007$, $\phi=.30$. The results of this initial experiment suggest that chasing is perceived much more robustly when the wolf and sheep are parsed as discrete objects rather than as parts of more global objects. In other words, an object chasing another object is perceived as such more readily than a line-end chasing another line-end.

\section{Experiment 2: objective detection}

The fact that observers used more mental state language to describe objects chasing objects (as opposed to line-ends chasing line-ends) suggests that the underlying units of perceived animacy may be objects - and this difference is consistent with the experiences readers may have when viewing the online demonstrations. However, descriptions are exceptionally poor at isolating particular mental processes. The observed difference could be due to a corresponding difference in perception, but also to higher level factors unrelated to visual processing, per se. For example, observers in the Connected condition may have detected the chasing relationship (among the line-ends) just as easily, but chosen not to describe it in animate terms because of the conceptual or linguistic awkwardness of reporting that a part of an object is chasing a part of another object. A more interesting possibility is that this influence was earlier and more automatic - that the descriptive difference was driven by a genuine perceptual difference: perhaps observers were more likely to fail to notice the

\section{Expt 1: Descriptions}

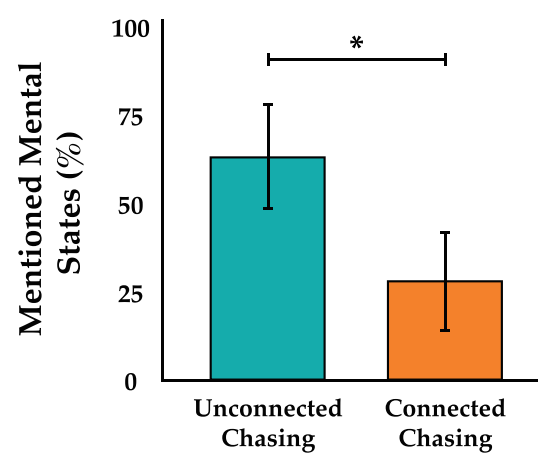

Fig. 2 Rates of mental state language use for subjects in the Unconnected and Connected conditions of Experiment 1. Error bars reflect $95 \%$ confidence intervals chasing relationship altogether when the wolf and sheep were both line-ends. To explore this possibility, we next tested whether this same disparity would manifest in the context not of subjective impressions but of objective performance: Would observers detect chasing less successfully in the Connected condition?

\section{Method}

Experiment 2 was identical to Experiment 1, except as noted here.

Observers Forty new observers participated in 30-min sessions in exchange for payment or course credit. This (withinsubjects) sample size matched the number of observers tested in each (between-subjects) condition in Experiment 1.

Stimuli Chasing-present displays were identical to those from Experiment 1, and since this was a detection task, we also included new chasing-absent displays. These were identical, except that the wolf updated its heading to approach a different target - not the sheep, but rather the reflection of the sheep's location through the display's vertical midline. We generated chasing-absent displays for both the Connected and Unconnected conditions. In both cases, because the wolf's target was not drawn, the displays did not evoke the impression of chasing (see Gao et al., 2009).

Procedure Observers were familiarized with the Unconnected chasing-present and Connected chasingpresent displays and were informed (a) that chasing would occur on half of trials, and (b) that chasing would be performed by line-ends as often as discs. On each trial, observers viewed a 4-s animation and were then immediately prompted to press one of two keys to indicate whether they had detected chasing - after which the next trial immediately began. Observers completed 84 practice trials ( 21 of each of the four trial types, in a different randomized order for each observer), the results of which were not recorded. They then completed 160 experimental trials, and were given breaks after the practice and halfway through the experimental trials.

\section{Results and discussion}

We categorized each response as a hit, miss, false alarm, or correct rejection and then computed $d^{\prime}$ (a measure of sensitivity, as distinct from response bias; Green \& Swets, 1966) for the Unconnected and Connected conditions. These $d^{\prime}$ scores are depicted in Fig. 3, and inspection of the figure indicates that observers were much more sensitive in the Unconnected condition $\left(d^{\prime}=1.65\right)$ compared to the Connected condition $\left(d^{\prime}=.99\right), t(39)=5.98, p<.001, d=$ .95 . These results indicate that chasing detection is object- 


\section{Expt 2: Detection}

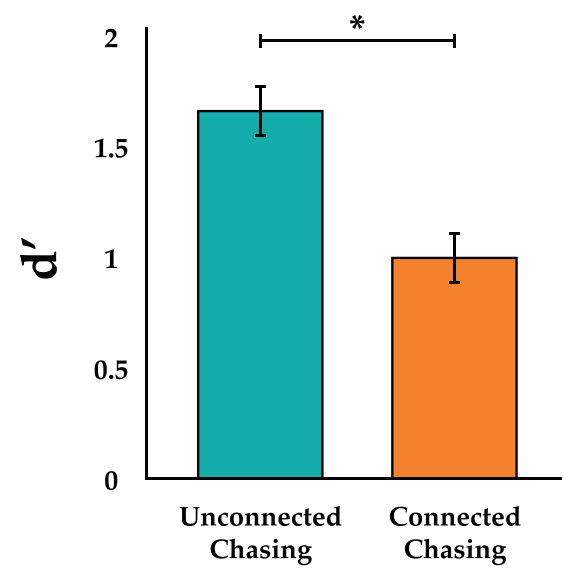

Fig. 3 Sensitivity ( $d^{\prime}$ values) for the Unconnected and Connected conditions of Experiment 2. Error bars reflect 95\% confidence intervals, subtracting out the shared variance

based not only in terms of visual phenomenology but also in terms of objective visual detection performance: Observers can readily detect an object chasing another object, but they are impaired at detecting a line-end chasing a line-end.

\section{Experiment 3: wolf versus sheep}

In the previous experiments, either both the wolf and sheep were discrete objects, or neither was. This leaves open the question of which of the "connections" was the culprit. In Experiment 2, for example, was the impaired sensitivity to chasing driven by the wolf's connection, the sheep's connection — or both? To find out, we replicated Experiment 2 while unconfounding these factors - essentially asking how readily observers can detect an object chasing a line-end, or a line-end chasing an object.

\section{Method}

This experiment was identical to Experiment 2 except as noted here. Forty new observers participated, twenty in each condition. (This sample size was chosen to be exactly half of that used in Experiment 2, based on the discovery from a power analysis that Experiment 2 was massively overpowered to find effects of the resulting magnitude.) In the Connected condition, one of the lines connected two distractors (as did both lines in the Unconnected condition). For half of observers (the wolf-disruption group), the other line connected the wolf to a distractor, and for the other half (the sheep-disruption group), the other line connected the sheep to a distractor.

\section{Results and discussion}

We computed $d^{\prime}$ for the Unconnected and Connected conditions for both groups of observers. These $d^{\prime}$ scores are depicted in Fig. 4, and inspection of the figure immediately indicates that observers in both groups were much less sensitive to Connected chasing. This impression was verified with a 2 (unconnected/connected) $\times 2$ (wolf-disruption group/ sheep-disruption group) analysis of variance, which revealed a significant effect of connectedness, $F(1,38)=55.78, p<$ $.001, \eta_{\mathrm{p}}{ }^{2}=.60$, but no effect of group, $F(1,38)=.02, p=.885$, $\eta_{\mathrm{p}}{ }^{2}<.01$, and no interaction, $F(1,38)=.57, p=.454, \eta_{\mathrm{p}}{ }^{2}=$ .02 . Sensitivity was higher in the Unconnected condition than in the Connected condition for both the wolf-disruption group (1.69 vs. 1.10$), t(19)=5.21, p<.001, d=1.16$, and the sheepdisruption group (1.61 vs. 1.13), $t(19)=5.44, p<.001, d=$ 1.22. These results reveal that $b o t h$ the wolf and sheep must be individuated as discrete objects in order for chasing detection to be maximally efficient.

\section{General discussion}

The three experiments reported here tell a consistent story: chasing detection is robust only when the "wolf" and "sheep" shapes are both parsed as discrete objects, and not when they are part of larger multielement objects. This was true both in terms of direct reports of phenomenology (Experiment 1) and in terms of the objective ability to detect chasing (Experiments 2 and 3). Critically, the impaired sensitivity to chasing with "line-ends" was observed even though this connectedness cue

\section{Expt 3: Selective Camouflage}

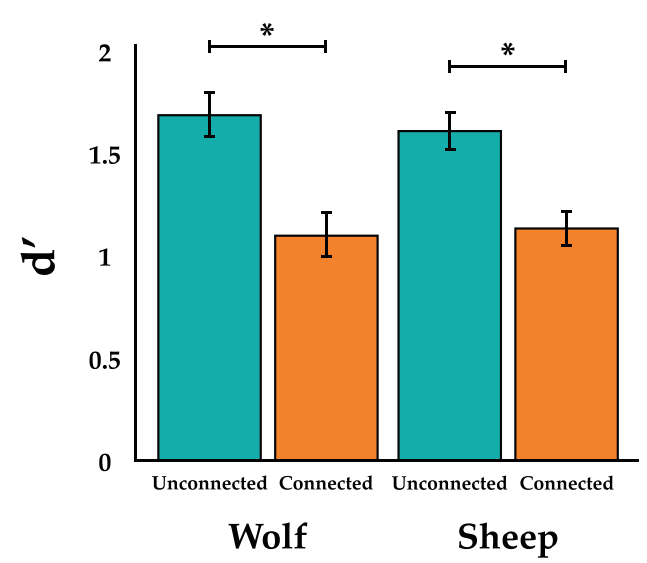

Fig. 4 Sensitivity ( $d^{\prime}$ values) for the Unconnected and Connected conditions for both groups of subjects in Experiment 3. For one group, the wolf was attached to a distractor in the Connected condition. For the other group, the sheep was attached to a distractor in the Connected condition. Error bars reflect $95 \%$ confidence intervals, subtracting out the shared variance 
was implemented with thin lines that left each shape readily discriminable (as in the figures and the online demonstrations).

These effects have three other properties that seem worth emphasizing. First, they were not just statistically robust, but also large - with Unconnected displays described 100\% more often than Connected displays in terms of mental state language (Experiment 1), and with chasing in Unconnected displays detected 67\% more readily than chasing in Connected displays in terms of $d^{\prime}$ values (Experiment 2). Second, the effects seem automatic, in the sense that they occurred (Experiments 2 and 3 ) even when observers were incentivized to ignore the lines and to see chasing as efficiently as possible even in Connected trials. Third, these effects seem highly specific, in that they cannot be attributed to any factors beyond object individuation. Critically, the thin lines were added to the display in a way that had no effect whatsoever on the motions or positions of the items; in other words, the Connected and Unconnected trials were entirely equated except for the differences in object individuation via connectedness.

Finally, these empirical results are consistent with the anecdotal experiences of observers who viewed the displays (as assessed by the reports collected in Experiment 1). For example, observers described Unconnected trials in terms of chasing (e.g., "The first circle chased the second") and evasion (e.g., "One seemed to be following the other, and it looked like the first was trying to evade the follower by bobbing/ weaving")-whereas observers described Connected trials in terms of asocial factors such as proximity (e.g., "The dots stayed the same distance apart") and similarity (e.g., "They moved in conjunction with each other around the square").

\section{Animacy and object-based attention?}

Our results suggest that the perception of chasing is objectbased, just as other processes such as attention seem to be. Might these be related? Previous work suggests that attention can be dynamically driven by perceived animacy, such that attention is automatically allocated to the wolf and sheep in a chasing display (Gao et al., 2017; see also Meyerhoff, Huff, \& Schwan, 2013). A more specific and intriguing possibility in the present context, though, is that the perception of chasing is object-based because attention is object-based.

In particular, detecting chasing may require the formation of a multiobject unit of attentional selection (i.e., a gestalt) consisting of a wolf and sheep. But the wolf and sheep may be groupable only if they are not already participating in other groups via possibly stronger cues, such as connectedness, so that disrupting the objecthood of both (Experiment 2) or either (Experiment 3) is sufficient to impair chasing detection. This possibility merits additional study but is consistent with reports that perceiving simple social relationships in very different contexts also involves grouping individual agents (Shen, Yin, Ding, Shui, \& Zhou, 2016). These results, however, are the first demonstration that such grouping may occur not only as an outcome of perceiving social relationships but also as a prerequisite for their detection.

\section{Conclusion: an ontological assumption in visual processing?}

Our results, in their most general form, signal the presence of a sort of "ontological assumption" in visual processing: It may be that before something can be seen as alive and goal directed, it must be individuated as a discrete "thing" in the first place.

Author note For helpful conversation and comments on previous drafts, we thank Greg McCarthy, Jamie McPartland, Hauke Meyerhoff, the members of the Yale Perception \& Cognition Laboratory, and two anonymous reviewers. This project was funded by an NSF Graduate Research Fellowship awarded to B.v.B., and by ONR MURI \#N0001416-1-2007 awarded to B.J.S.

\section{References}

Barrett, H., Todd, P., Miller, F., \& Blythe, M. (2005). Accurate judgments of intention from motion cues alone: A cross-cultural study. Evolution and Human Behavior, 26, 313-331.

Cave, K. R., \& Bichot, N. P. (1999). Visuospatial attention: Beyond a spotlight model. Psychonomic Bulletin and Review, 6, 204-223.

Chen, Z. (2012). Object-based attention: A tutorial review. Attention, Perception and Psychophysics, 74, 784-802.

Csibra, G. (2008). Goal attribution to inanimate agents by 6.5-month-old infants. Cognition, 107, 705-717.

Dittrich, W., \& Lea, S. (1994). Visual perception of intentional motion. Perception, 23, 253-268.

Egly, R., Driver, J., \& Rafal, R. (1994). Shifting visual attention between objects and locations: Evidence from normal and parietal lesion subjects. Journal of Experimental Psychology: General, 123, 161177.

Feldman, J. (2007). Formation of visual "objects" in the early computation of spatial relations. Perception and Psychophysics, 69, 816827.

Fornaciai, M., Cicchini, G. M., \& Burr, D. C. (2016). Adaptation to number operates on perceived rather than physical numerosity. Cognition, 151, 63-67.

Franconeri, S. L., Bemis, D. K., \& Alvarez, G. A. (2009). Number estimation relies on a set of segmented objects. Cognition, 113, 1-13.

Gao, T., McCarthy, G., \& Scholl, B. J. (2010). The wolfpack effect: Perception of animacy irresistibly influences interactive behavior. Psychological Science, 21, 1845-1853.

Gao, T., New, J. J., \& Scholl, B. J. (2017). The wavering wolf: Perceived intentionality controls attentive tracking. Manuscript submitted for publication.

Gao, T., Newman, G. E., \& Scholl, B. J. (2009). The psychophysics of chasing: A case study in the perception of animacy. Cognitive Psychology, 59, 154-179.

Gao, T., \& Scholl, B. J. (2011). Chasing vs. stalking: Interrupting the perception of animacy. Journal of Experimental Psychology: Human Perception and Performance, 37, 669-684.

Garfield, P. (2001). Universal dream key: The 12 most common dream themes around the world. New York, NY: HarperCollins. 
Green, D., \& Swets, J. (1966). Signal detection theory and psychophysics. New York, NY: Wiley.

He, L., Zhang, J., Zhou, T., \& Chen, L. (2009). Connectedness affects dot numerosity judgment: Implications for configural processing. Psychonomic Bulletin and Review, 16, 509-517.

Heider, F., \& Simmel, M. (1944). An experimental study of apparent behavior. American Journal of Psychology, 57, 243-259.

Howe, P. D., Incledon, N. C., \& Little, D. R. (2012). Can attention be confined to just part of a moving object? Revisiting target-distractor merging in multiple object tracking. PLOS ONE, 7, e41491.

Luck, S., \& Vogel, E. (1997). The capacity of visual working memory for features and conjunctions. Nature, 390, 279-281.

Luck, S., \& Vogel, E. (2013). Visual working memory capacity: From psychophysics and neurobiology to individual differences. Trends in Cognitive Science, 17, 391-400.

Marino, A. C., \& Scholl, B. J. (2005). The role of closure in defining the "objects" of object-based attention. Perception and Psychophysics, 67, 1140-1149.

Meyerhoff, H. S., Huff, M., \& Schwan, S. (2013). Linking perceptual animacy to attention: Evidence from the chasing detection paradigm. Journal of Experimental Psychology: Human Perception and Performance, 39, 1003-1015.

Meyerhoff, H. S., Schwan, S., \& Huff, M. (2014). Perceptual animacy: Visual search for chasing objects among distractors. Journal of Experimental Psychology: Human Perception and Performance, 40, 702-717.

Michotte, A. (1950-1991). The emotions regarded as functional connections. In M. Reymert (Ed.), Feelings and emotions: The Mooseheart symposium (pp. 114-125). New York: McGraw-Hill. Reprinted in Thinès, G., Costall, A., and Butterworth, G. (Eds.), Michotte's experimental phenomenology of perception (pp. 103-116). Hillsdale, NJ: Erlbaum, 1991.

Peirce, J. W. (2007). PsychoPy-Psychophysics software in Python. Journal of Neuroscience Methods, 162, 8-13.
Revonsuo, A. (2000). The reinterpretation of dreams: An evolutionary hypothesis of the function of dreaming. Behavioral and Brian Sciences, 23, 793-1121.

Rochat, P., Morgan, R., \& Carpenter, M. (1997). Young infants' sensitivity to movement information specifying social causality. Cognitive Development, 12, 537-561.

Scholl, B. J. (2001). Objects and attention: The state of the art. Cognition, $80,1-46$.

Scholl, B. J., \& Gao, T. (2013). Perceiving animacy and intentionality: Visual processing or higher-level judgment? In M. D. Rutherford \& V. A. Kuhlmeier (Eds.), Social perception: Detection and interpretation of animacy, agency, and intention (pp. 197-230). Cambridge, MA: MIT Press.

Scholl, B. J., Pylyshyn, Z. W., \& Feldman, J. (2001). What is a visual object? Evidence from target merging in multi-element tracking. Cognition, 80, 159-177.

Scholl, B. J., \& Tremoulet, P. (2000). Perceptual causality and animacy. Trends in Cognitive Sciences, 4, 299-309.

Shen, M., Yin, J., Ding, X., Shui, R., \& Zhou, J. (2016). Deployment of attention on handshakes. Frontiers in Psychology, 7, 1-12.

Suchow, J. W., Fougnie, D., Brady, T. F., \& Alvarez, G. A. (2014). Terms of the debate on the format and structure of visual memory. Attention, Perception and Psychophysics, 76, 2071-2079.

van Buren, B., \& Scholl, B. J. (in press). Minds in motion in memory: Enhanced spatial memory driven by the perceived animacy of simple shapes. Cognition.

van Buren, B., Uddenberg, S., \& Scholl, B. J. (2016). The automaticity of perceiving animacy: Goal-directed motion in simple shapes influences visuomotor behavior even when task-irrelevant. Psychonomic Bulletin and Review, 23, 797-802.

Vogel, E. K., Woodman, G. F., \& Luck, S. J. (2001). Storage of features, objects, and conjunctions in working memory. Journal of Experimental Psychology: Human Perception and Performance, 27, 92-114. 